\title{
SMD Simulations of Shear Loading Induced Dissociation of P-selectin/PSGL-1 Complex
}

\author{
Peng Ren, Shouqin Lü, Yingyong Kang, Bo Huo, \\ Mian Long*
}

\begin{abstract}
Selectin/ligand interactions mediate the tethering and rolling processes of blood cells on vascular surfaces. It has been demonstrated that flow is required during this adhesion process. But the molecular mechanism, e.g. how the shear loading from the hydrodynamic environment of the circulation influences the rupture of selectin/ligand complex, is still unclear. In this study, we simulated the dissociation process of P-selectin/PSGL-1 complex under shear loading at the atomic level. The shear field was established by stretching the top layer of water molecules using SMD method. The dynamic evolvement of microstructure of P-LE/SGP-3 complex, the minimal functional unit of Pselectin/PSGL-1 complex, for different shear velocity was studied. The simulation results indicated that the extension in PLE occurred before the dissociation of P-LE/SGP-3 complex. During the dissociation process, two anti-parallel $\beta$-sheets in EGF and hydrogen bonds at the interface of EGF-Lec domains were broken, but the structure of Lec domain remained unchanged. Under the same initial inclination angle of molecular complex relative to flow direction, structural destruction time decreased but dissociation speed of the complex increased along with the increase of shear velocity. For the same shear velocity, the dissociation of the complex was speeded up when decreasing the initial inclination angle. This work provide a insight into understand the structural bases for the rupture of $P$ selectin/PSGL-1 bond at atomic level.
\end{abstract}

Keywords-SMD; selectin; adhesion; molecular interaction

\section{INTRODUCTION}

Selectin/ligand interactions mediate the tethering and rolling processes of blood cells on vascular surfaces, a prerequisite for inflammatory cascade or tumor metastases [1]. P-selectin, one of members of selectin family, is expressed on activated endothelial cells and its high-affinity ligand, PSGL-1 (P-selectin glycoprotein ligand 1), on leukocytes. P-selectin consists of an N-terminal, calcium-type lectin (Lec) domain, an epidermal growth factor (EGF)-like domain, followed by nine copies of consensus repeat (CR) units [2]. Selectin interacts with PSGL-1 by forming a binding pocket containing Lec-EGF (LE) domains of selectin and specific sulfoglycopeptide of PSGL-1. This is exemplified as a X-ray crystallographic structure of P-LE/SGP-3 complex [3], in which SGP-3 is a synthesized sulfoglycopeptide and the minimal functional unit of wild type PSGL-1 molecule [4].

Selectin/ligand interactions are modulated by external forces [5]. Under physiological condition, selectin interacts with PSGL-1 in two-dimension since both molecules are anchored on the surfaces of two apposing cells. This results in that their interactions are tightly coupled with mechanical

\author{
National Microgravity Laboratory and Center for \\ Biomechanics and Bioengineering, Institute of Mechanics, \\ Chinese Academy of Sciences, Beijing, China \\ *Corresponding author (mlong@imech.ac.cn)
}

environment of blood flow, which translates into an external force for modulating the dissociation of selectin/PSGL-1 bond [6]. To mediate leukocyte rolling in blood stream, dissociation of selectin/PSGL-1 bond in the rear should balance with bond formation in the front. This turns out to stretch the bond complex in the rear until it breaks up. It has been demonstrated that flow is required during this adhesion process [7]. It is easily imagined that another type of force, shear loading from the hydrodynamic environment of the circulation, may also play a role on the dissociation of P-selecin/PSGL-1 complex.

Steered molecular dynamics (SMD) simulation is a well developed approach to conduct the simulations of force induced molecular unfolding and dissociation by introducing external forces into molecular dynamics simulations [8], which can provide the time-dependent conformational changes at atomic level on the time scale accessible to molecular dynamic simulations. We have numerically simulated the dissociation process of P-selectin/PSGL-1 complex under stretching force with steered molecular simulation (SMD) method [9]. The present work further focused on the effect of shear loading rate and initial inclination angle of molecular complex on the dynamic evolvement of conformation of the complex.

\section{MATERIALS AND METHODS}

Similar to our previous work [9], SMD simulations were performed using the program of NAMD2 with CHARMM22 force field, plus the program of VMD for hydrogen bond analyses and structural visualizations. Using SOLVATE1.2, the plug-in of VMD, we set up a cuboid pure water box, which is about $196 \AA$ long, $67 \AA$ wide and $131 \AA$ high including 164895 atoms (Figure 1), for testing the feasibility of SMD method in shear flow building. X-axis is along the flow direction and the velocity of $v_{\mathrm{x}}$ was assigned to vary linearly along Y-axis. TIP3P water model was used in the empirical potential. By stretching the top layer of water molecules of $5 \AA$ height using $\mathrm{c} v$-SMD, and fixing the bottom $5 \AA$ layer in $\mathrm{Y}$ direction, the shear flow with linear gradient field of velocity $v_{\mathrm{x}}(y)=\mathrm{k} y$ was expected to be produced via the diffusion of water molecules along $\mathrm{Y}$ direction and molecular interaction between different layers.

P-LE/SGP-3 complex, similar to our previous study $[9,10]$, was chosen to investigate its conformational evolvement in shear flow. Molecular complex was solvated in a cuboid water box with the dimensions of $141 \times 111 \times 72 \AA^{3}$ (Figure 1). The D158- $\mathrm{C}_{\alpha}$ atom at C-terminal of EGF domain was fixed at bottom surface. The complex inclined as an angle of $30^{\circ}$ or $60^{\circ}$ from the flow direction, i.e. between $\mathrm{X}$-axis and the line 
connecting D158- $\mathrm{C}_{\alpha}$ of EGF and P618- $\mathrm{C}_{\alpha}$ of SGP-3 peptide. After system neutralization, energy minimization, and equilibration, the water molecules in the top layer with $10 \AA$ thickness were initially assigned as the velocities of $0.5,1$ and $5 \AA / \mathrm{ps}$, and performed simulations for 3000,1000 and 250 ps respectively. Bottom layer with $20 \AA$ thickness was fixed and periodic boundary condition was adopted.

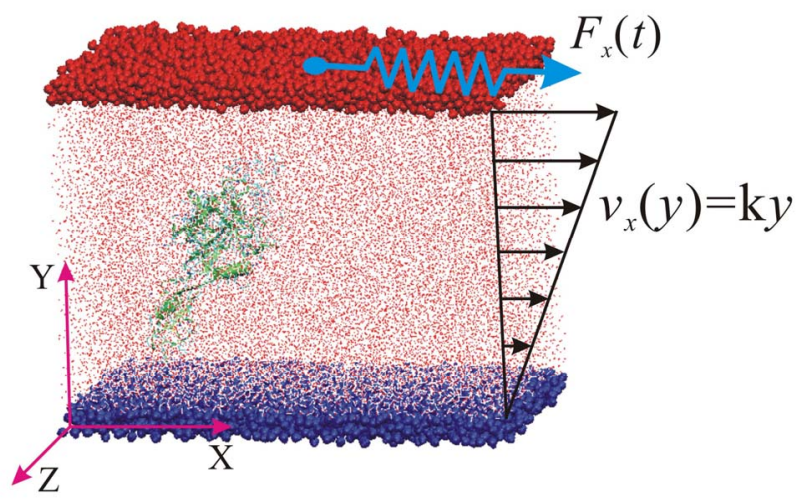

Figure 1 A water cuboid box in which the bottom layer (blue) was fixed and the top layer (red) was stretched by $\mathrm{c} v$-SMD to form a linear gradient field of velocity $v_{\mathrm{x}}(y)$. The P-LE/SGP-3 system was placed in this water box (water molecules were presented as lines, P-LE as cartoon(cyan), peptide in SGP-3 as cartoon (red), glycosides in SGP-3 as bonds (yellow), and $\mathrm{Ca}^{2+}$ as VDW (silver)).

To quantitatively describe the dynamic changes of conformation of P-LE/SGP-3 complex under shear loading, some characteristic parameters were defined (Figure 2). $\triangle \mathrm{x}$ denotes the distance between $\mathrm{P} 618-\mathrm{C}_{\alpha}$ of SGP-3 peptide and D158- $\mathrm{C}_{\alpha}$ of EGF domain showing the extension of complex. $\triangle y$ indicates the distance between the centers of Lec domain and SGP-3 showing the dissociation of complex. D1 is the distance between the center of the two primary anti-parallel $\beta$ sheets (G131-E135 and Y140-C144) in EGF, which is to reveal the broken level of main anti-parallel $\beta$-sheet. D2 denotes the distance between $\mathrm{T} 2-\mathrm{C}_{\alpha}$ and $\mathrm{I} 137-\mathrm{C}_{\alpha}$ in P-LE showing the broken level of Lec-EGF interface. D3 is the distance between $\mathrm{C}_{\mathrm{a}}{ }^{2+}$ ion and the center of FUC (Fucose, a residue of core 2branched O-glycan in SGP-3) showing the end of complex dissociation. A1 is the angle of the line connecting P618- $\mathrm{C}_{\alpha}$ in SGP-3 and D158- $\mathrm{C}_{\alpha}$ in EGF relative to XZ plane showing the angle of complex in shear flow. $\mathrm{H} 1$ denotes the number of hydrogen bonds in the primary anti-parallel $\beta$-sheet of EGF (S121-D158). H2 is the number of hydrogen bonds in the interface of Lec domain (W1-A120) and EGF domain (S121D158). H3 is the number of hydrogen bonds between Lec domain (W1-A120) and peptide in SGP-3. H4 is the number of hydrogen bonds between Lec domain (W1-A120) and glycoside in SGP-3. RMSD of Lec domain (W1-A120) and EGF domain (S121-D158) showing the conformational breakage of each functional domain.

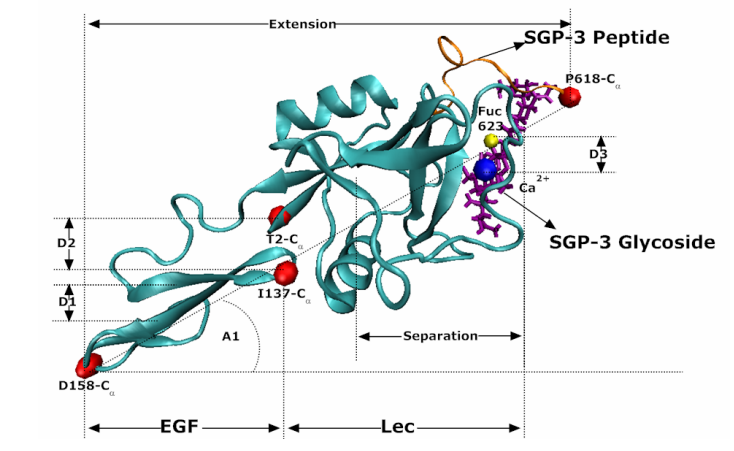

Figure 2 Definations of characteristic parameters for conformational description of P-LE/SGP-3 complex.

\section{RESULTS AND DISCUSSIONS}

The simulations of pure water system showed that for the different loading rate of 1 or $5 \AA / p s$, an obvious linear velocity gradient was formed since $100-150$ ps and gradually became stable at 200-250 ps (data not shown). It has been confirmed that the shear flow could be formed under SMD method and that the velocity gradient in flow is reasonable to study the dynamic variation of microscopic structure of the complex under shear loading.

As a typical simulation of water-molecule system with loading rate of $1 \AA / \mathrm{ps}$ and initial inclination angle of $30^{\circ}$, the dissociation process of the complex was quantitatively analyzed. Figure $3 a-d$ show some characteristics of the variation of microscopic structure of the molecular complex under shear loading and Figure $3 e$ shows typical snapshots of the complex during dissociation process. It was easily seen that during dissociation of the complex, the "separation" of selectin/ligand binding pocket occurred much later than the "extension" of whole P-LE/SGP-3 complex (Figure 3a) and then mainly contributed to the deformation of the complex when the "extension" reached about $115 \AA$. Integrating with Figure $3 e$, the "extension" before separation mainly occurred inside the P-LE, especially the EGF domain and Lec-EGF interface. This conclusion was reconfirmed by the fact that the RMSD of EGF domain first rapidly increase to a platform of $11 \AA$, while that of Lec domain only changed by $4 \AA$ (Figure $3 b$ ). During the breakage process of EGF domain, the antiparallel $\beta$-sheets collapsed. As shown in Figure $3 c$, the collapse of anti-parallel $\beta$-sheets in EGF domain and the breakage of EGF-Lec interface occurred at 200-300 ps after the onset of shear loading, which is much earlier than the deforming of Lec domain and the separating of P-LE/SGP-3 complex occurring at around $600 \mathrm{ps}$ (Figure $3 d$ ). The fact that the number of hydrogen bonds $\mathrm{H} 3$ decreased to zero a little bit early than $\mathrm{H} 4$ implies that on the P-LE/SGP-3 interface, destruction occurs first on the hydrogen bonds between Lec domain and the peptide of SGP-3 and then on the hydrogen bonds between the Lec domain and the glycoside of SGP-3. 

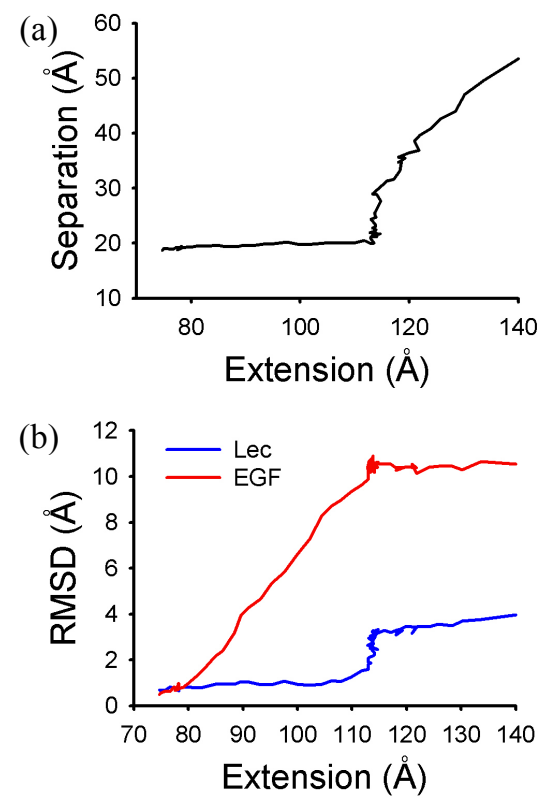

(e)

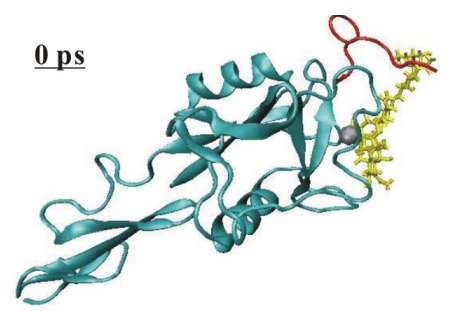

(c)
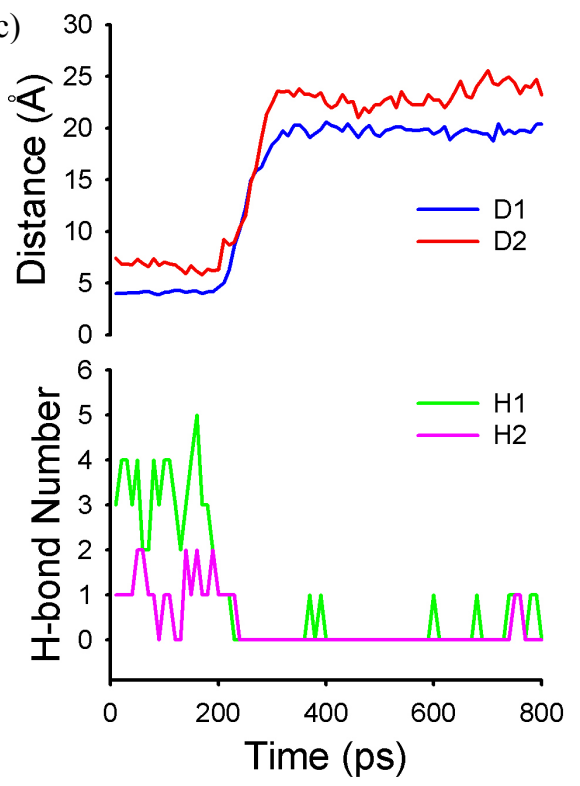
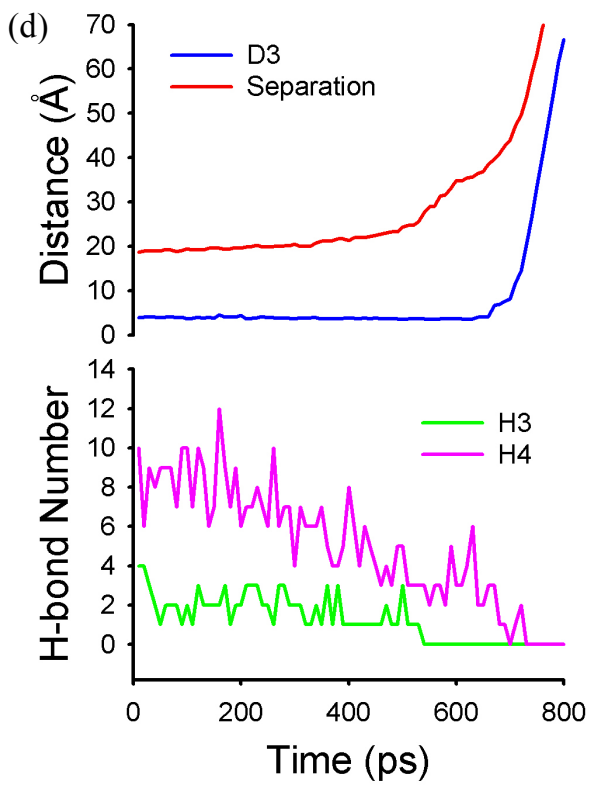
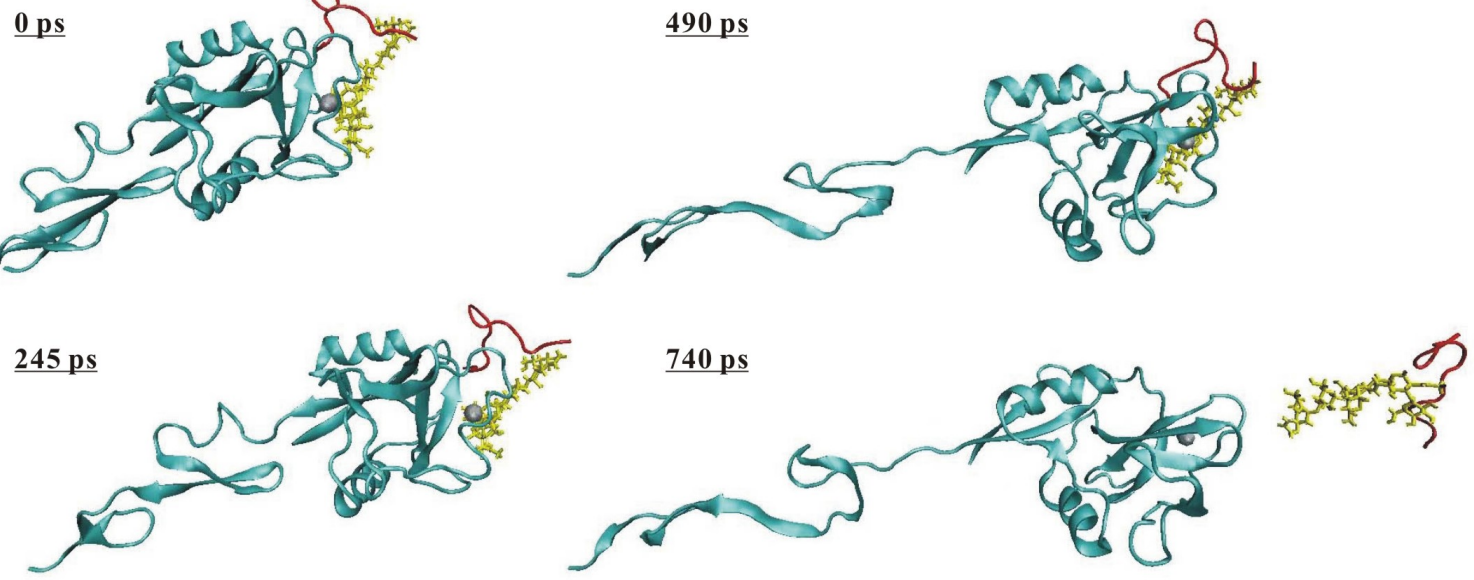

Figure 3 Some characterisitics during the dissociation of P-LE/SGP-3 complex for shear loading rate of $1 \AA / \mathrm{ps}$ and initial inclination angle of 30 degree (a-d) and snapshots of molecular sturcture at four time points, in which P-LE is presented as cartoon(cyan), peptide in SGP-3 as cartoon (red), glycosides in SGP-3 as bonds (yellow), and $\mathrm{Ca}^{2+}$ as VDW (silver).

For the catch bond phenomenon of selectin-ligand interaction, i.e. bond lifetime of selectin-PSGL-1 interactions is prolonged with applied forces [6], a possible explanation is that the opening of the hinge between Lec and EGF domains leads to a sliding-rebinding of binding interface of P-LE/SGP3 [11]. Our present study also revealed the opening of hinge, e.g. the hinge angle increased from 140 to 160 degree for shear loading rate of $1 \AA / \mathrm{ps}$, as well as the breaking and reforming of hydrogen bonds on the interacting interface between Lec domain and the peptide or glycoside of SGP-3 (Figure 4). Under the application of shear loading, the inclination angle of molecular complex relative to flow direction gradually decreased from 30 to 18 degree. This inclination towards flow direction resulted in that the interface between P-LE and SGP3 gradually became vertical to the flow direction, which is believed to speed up the dissociation of the complex.
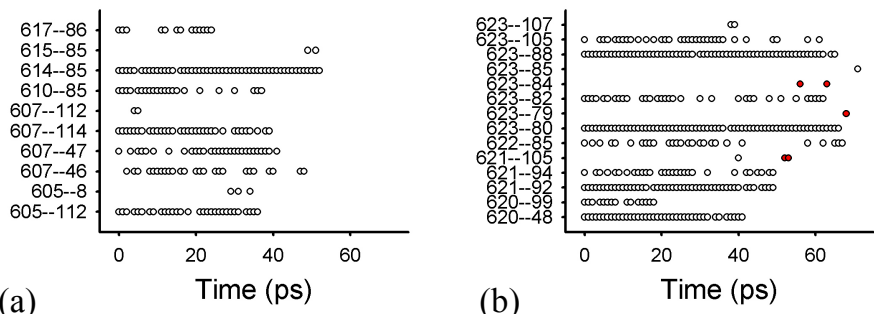

Figure 4 The change of hydrogen bonds during the dissociation of $\mathrm{P}$ LE/SGP-3 complex for shear loading rate of $1 \AA / \mathrm{ps}$. Hydrogen bonds between peptide in SGP-3 and Lec domain a); and between glycosides in SGP-3 and Lec domain b).

For the case of the initial inclination angle of 30 degree, we compared the dissociation processes under three loading rates of $0.5,1$ and $5 \AA / \mathrm{ps}$. As we described above, main intramolecular destruction of the complex is in EGF domain, so RMSD of EGF domain was used to indicate the 
conformational change of the complex. Figure $5 a$ shows that along with the increase of shear loading rate, the occurring of conformational change becomes significantly early. The increase of shear loading rate also speeded up the dissociation of the complex. For different loading rate, the hinge angle of Lec-EGF remains similar. The final inclination angle of the complex towards flow direction decreased with the increase of loading rate (Figure $5 b$ ).

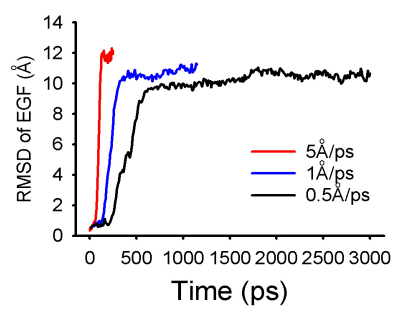

(a)

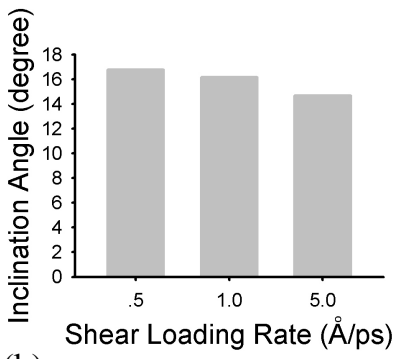

(b)
Figure 5 RMSD (a) and final inclination angle (b) of P-LE/SGP-3 complex for different shear loading rates of 5,1 and $0.5 \AA / p s$ respectively, and the same initial inclination angle of 30 degree,

We also considered another initial inclination angle of 60 degree under the same shear loading rate of $5 \AA / \mathrm{ps}$. Compared with the results for 30 degree, P-LE/SGP-3 complex for 60 degree was separated more quickly with more extension of whole molecule complex (Figure $6 a$ ). It was also shown that the larger initial inclination angle increased $\mathrm{A} 1$, the final angle of the line connecting P618- $\mathrm{C}_{\alpha}$ in SGP-3 and D158- $\mathrm{C}_{\alpha}$ in EGF relative to $\mathrm{XZ}$ plane (Figure $6 \mathrm{~b}$ ). These results are consistent with the above discussions on Figure 4, i.e. the inclination towards flow direction may speed up the dissociation of the complex. For both initial inclination angles, no other significant difference was found in other characteristics such as RMSD of EGF, the extension of the complex and the hinge angles at the end of dissociation process.

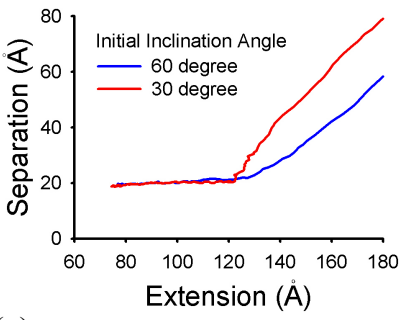

(a)

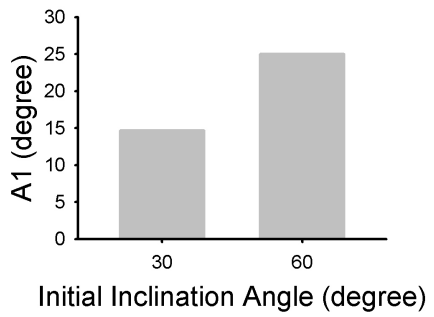

(b)
Figure 6 The effect of initial inclination angles of 60 and 30 degree under shear loading rate of $5 \AA$ ps on (a) RMSD of EGF domain and (b) final angle of A1.

\section{CONCLUSION}

This preliminary work successfully established a reasonable flow field and studied the dynamic evolvement process of microscopic structure of P-LE/SGP-3 complex, the minimal functional unit of P-selectin/PSGL-1 complex, under shear loading. It was demonstrated in the present study that the complex was able to dissociate even only under shear loading. The dynamic evolvement processes of atomic bonds, secondary structures and molecular deformation were analyzed for three different flow velocities. There were some common characters on the structural evolvements of the selectin-ligand complex regardless of the varying flow velocities. The molecular extension was prior to the complex's dissociation, which is characterized by the separation of fucose unit (FUC) from calcium ion. Before the dissociation, the rupture of secondary structures in EGF occurred earlier than LEC. Shear loading resulted in the decrease of the inclination angle of the complex relative to the flow direction and the increase of hinge angle between EGF and LEC. Along with the increase of flow velocity, the molecular extension and the dissociation of the complex were accelerated. The latter also took place when increasing the initial inclination angle of the complex.

\section{ACKNOWLEDGEMENTS}

This work was supported by National Key Basic Research Foundation of China grant 2006CB910303, National Natural Science Foundation of China grants 10702075, 30730032, and 10332060, 30300076, and National High Technology Research and Development Program of China grant 2007AA02Z306, and Innovation grant, Institute of Mechanics, Chinese Academy of Sciences.

\section{REFERENCES}

[1] T. A. Springer, "Adhesion receptors of the immune system," Nature, vol. 346, pp. 425-434, Aug 21990.

[2] K. Ley, P. Gaehtgens, C. Fennie, M. S. Singer, L. A. Lasky et al.,, "Lectin-like cell adhesion molecule 1 mediates leukocyte rolling in mesenteric venules in vivo," Blood, vol. 77, pp. 2553-2555, Jun 15 1991.

[3] W. S. Somers, J. Tang, G. D. Shaw, and R. T. Camphausen, "Insights into the molecular basis of leukocyte tethering and rolling revealed by structures of P- and E-selectin bound to SLe(X) and PSGL-1," Cell, vol. 103, pp. 467-479, Oct 272000.

[4] A. Leppanen, P. Mehta, Y. B. Ouyang, T. Ju, J. Helin et al.,, "A novel glycosulfopeptide binds to P-selectin and inhibits leukocyte adhesion to P-selectin," J Biol Chem, vol. 274, pp. 24838-24848, Aug 271999.

[5] S. Q. Lu, Z. Y. Ye, C. Zhu, and M. Long, "Quantifying the effects of contact duration, loading rate, and approach velocity on P-selectinPSGL-1 interactions using AFM," Polymer, vol. 47, pp. 2539-2547, Mar 222006.

[6] B. T. Marshall, M. Long, J. W. Piper, T. Yago, R. P. McEver et al., "Direct observation of catch bonds involving cell-adhesion molecules," Nature, vol. 423, pp. 190-193, May 82003.

[7] C. Zhu, T. Yago, J. Z. Lou, V. I. Zarnitsyna, and R. P. McEver, "Mechanisms for flow-enhanced cell adhesion," Annals of Biomedical Engineering, vol. 36, pp. 604-621, Apr 2008.

[8] B. Isralewitz, M. Gao, and K. Schulten, "Steered molecular dynamics and mechanical functions of proteins," Curr Opin Struct Biol, vol. 11, pp. 224-230, Apr 2001.

[9] S. Lu and M. Long, "Forced dissociation of selectin-ligand complexes using steered molecular dynamics simulation," Mol Cell Biomech, vol. 2, pp. 161-177, Dec 2005.

[10] S. Q. Lu and M. Long, "Forced extension of P-selectin construct using steered molecular dynamics," Chinese Science Bulletin, vol. 49, pp. 1017, Jan 2004.

[11] J. Lou, T. Yago, A. G. Klopocki, P. Mehta, W. Chen et al.,, "Flowenhanced adhesion regulated by a selectin interdomain hinge," J Cell Biol, vol. 174, pp. 1107-1117, Sep 252006. 